\title{
Modeling and manufacturability assessment of bistable quantum-dot cells
}

\author{
M. Governale, M. Macucci, ${ }^{\text {a) }}$ G. lannaccone, and C. Ungarellib) \\ Dipartimento di Ingegneria dell'Informazione, Università di Pisa, Via Diotisalvi, 2, \\ I-56126 Pisa, Italy \\ J. Martorell \\ Departament d'Estructura i Constituents de la Materia, Facultat de Física, Universitat de Barcelona, \\ Barcelona 08028, Spain
}

(Received 24 April 1998; accepted for publication 10 November 1998)

\begin{abstract}
We have investigated the behavior of bistable cells made up of four quantum dots and occupied by two electrons, in the presence of realistic confinement potentials produced by depletion gates on top of a GaAs/AlGaAs heterostructure. Such a cell represents the basic building block for logic architectures based on the concept of quantum cellular automata (QCA) and of ground state computation, which have been proposed as an alternative to traditional transistor-based logic circuits. We have focused on the robustness of the operation of such cells with respect to asymmetries derived from fabrication tolerances. We have developed a two-dimensional model for the calculation of the electron density in a driven cell in response to the polarization state of a driver cell. Our method is based on the one-shot configuration-interaction technique, adapted from molecular chemistry. From the results of our simulations, we conclude that an implementation of QCA logic based on simple "hole arrays" is not feasible, because of the extreme sensitivity to fabrication tolerances. As an alternative, we propose cells defined by multiple gates, where geometrical asymmetries can be compensated for by adjusting the bias voltages. Even though not immediately applicable to the implementation of logic gates and not suitable for large scale integration, the proposed cell layout should allow an experimental demonstration of a chain of QCA cells. (C) 1999 American Institute of Physics. [S0021-8979(99)04004-9]
\end{abstract}

\section{INTRODUCTION}

Several proposals for the implementation of logic functions and data processing based on the concept of quantum cellular automata (QCA) and ground state computation have appeared ${ }^{1-4}$ in the literature recently. Tougaw et al. ${ }^{1}$ devised an architecture (commonly known as "Notre Dame architecture") based on bistable cells that couple electrostatically to their nearest neighbors. Each cell consists of four (or five) quantum dots and contains two electrons. In the absence of external electric fields, the electrons occupy each dot with equal probability. In the presence of a nearby (driver) cell with the two electrons constrained to occupy the dots along one of the two diagonals, alignment along the parallel diagonal will occur in the driven cell, in the hypothesis of potential barriers large enough to localize the electrons. Based on this principle, it is possible to conceive of bistable cell arrays, in which the polarization state enforced at the inputs, at the edges of the arrays, propagates in a "domino" fashion ${ }^{5}$ until the ground state is reached throughout the system, and the results of the computation are available in the form of the polarization state of the output cells, also located at the edges of the arrays.

Many issues must be resolved before this computational paradigm can be implemented in practice: noninvasive detectors are needed to probe the polarization state of the outputs

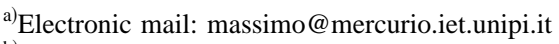

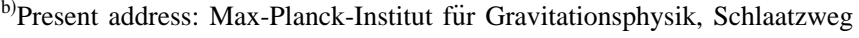
1, D-14473 Potsdam, Germany.
}

without perturbing the ground state of the system; provision must be made for a time evolution of the system that is both fast and reliable; design solutions for the basic cell must be developed, yielding a reasonable robustness to fabrication tolerances and compatible with large-scale integration on a single chip.

The focus of the work we are presenting is specifically on the robustness of a single cell, coupled to a driver cell, to fabrication tolerances and to asymmetries caused by fluctuations in the bias voltages applied to the electrodes defining the cell. In particular, we have studied the effect of geometrical and electrical asymmetries on the behavior of QCA cells defined by means of lateral electrostatic confinement in a GaAs/AlGaAs heterostructure.

Although often neglected, robustness to fabrication tolerances and manufacturability are central problems affecting all proposed nanoelectronic devices, ${ }^{6}$ and their solution is a prerequisite for any successful new technology.

We have considered a basic QCA cell with four quantum dots defined by realistic two-dimensional (2D) confinement potentials, which are computed from the shape of the metal gates at the surface of the heterostructure and the voltages applied to them. Calculation of the electron density in such a 2D artificial molecule is a challenging task. Iterative selfconsistent methods fail to converge, due to the relatively large electrostatic interaction and to the particular symmetries associated with the problem. For this reason, we have developed a noniterative technique based on the configuration-interaction (CI) method used in molecular 

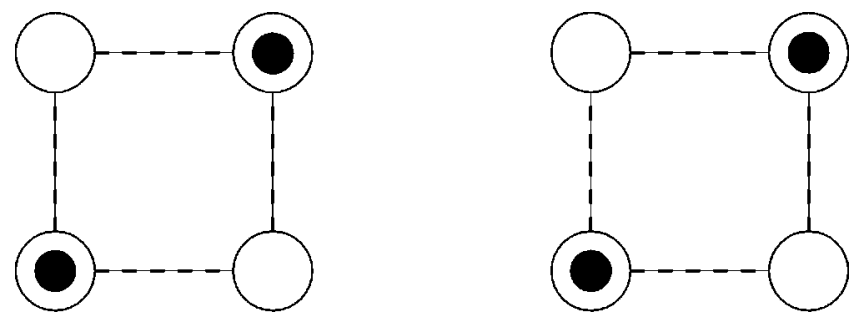

FIG. 1. Schematic representation of two coupled QCA cells: tunneling of electrons is possible along the dashed lines.

chemistry. ${ }^{7}$ The main drawback of this technique is that it requires rather large computational resources and, thus, we are presenting numerical results only for the case of occupancy of two electrons per cell. (Work is currently in progress for the inclusion of up to six electrons per cell.)

With respect to the approaches in the literature, ${ }^{8}$ our method allows a direct quantitative estimate of the effects of fabrication and bias tolerances on cell operation and does not require the introduction of phenomenological parameters such as the tunneling energy, which may be hard to evaluate with a realistic potential.

In Sec. II, we provide a detailed statement of the problem we intend to solve and describe the cell model together with the technique we have used for the computation of the 2D confinement potential. In Sec. III, the solution of the many-body problem is discussed and the one-shot CI method is introduced. Numerical results for a cell occupancy of two electrons and various types of asymmetries are presented in Sec. IV, where cell design criteria are also established.

\section{STATEMENT OF THE PROBLEM AND CELL MODEL}

Our aim is to investigate the behavior of two coupled QCA cells, each of which is formed by four quantum dots and contains two electrons, as illustrated in Fig. 1. Tunneling between the dots of the same cell is allowed, but not between dots belonging to different cells. If the barriers separating the dots within a cell are opaque enough, the electron wave functions will be localized, and we shall observe a quasiclassical behavior: the two electrons will repel each other and localize in two dots along a diagonal, so as to minimize the electrostatic energy. In the case of an isolated, symmetric cell, alignment along either diagonal will occur with equal probability. If another (driver) cell is placed in proximity to the cell we are investigating (driven cell), as in the case represented in Fig. 1, and the electrons in the driver cell are taken to be aligned along a given diagonal, their electric field will destroy the symmetry of the driven cell, lifting the degeneracy between the two configurations. This will result in the electrons in the driven cell lining up parallel to the electrons in the driver cell.

Following Lent $e t a .^{2}$ we define a cell polarization $P$ as

$$
P \equiv \frac{\left(Q_{1}+Q_{3}\right)-\left(Q_{2}+Q_{4}\right)}{Q_{1}+Q_{2}+Q_{3}+Q_{4}},
$$

where $Q_{i}$ is the integral of the electron density $\rho(\mathbf{r})$ over the $i$ th quadrant of a cell. We divide each cell into four quadrants (see Fig. 2) and number them counterclockwise. The

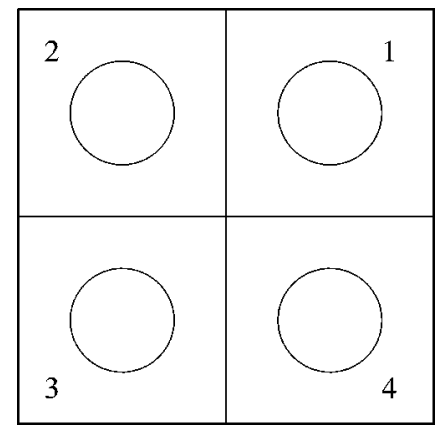

FIG. 2. Subdivision of a cell into four quadrants.

denominator of Eq. (1) is the total number of electrons in the cell and therefore a constant. The procedure for calculating the electron density from the many-electron wave functions will be discussed in Sec. III.

If the two electrons are aligned along the diagonal corresponding to the first and third quadrant, $P=1$, while if they are aligned along the other diagonal, $P=-1$. For finite height barriers, values of $P$ intermediate between -1 and 1 are also possible. We define the cell-to-cell response function as the function relating the polarization of the driven cell to that of the driver cell. In order to compute the polarization of the driven cell in response to each value of the polarization of the driver cell, we need to solve for the electronic structure in the driven cell in the presence of the electrostatic potential due to the driver cell.

For the driven cell we consider a 2D model in the effective mass approximation. Such a model is valid as long as the thickness of the dots in the vertical direction, corresponding to the thickness of the 2D electron gas (2DEG) from which they are obtained by lateral confinement, is small compared to their other dimensions. The 2DEG is obtained by modulation doping next to a GaAs/AlGaAs heterointerface.

The 2D confinement potential in the plane of the 2DEG is obtained as a result of the action of the metal gates, following the method proposed by Davies et al., ${ }^{9,10}$ without including (to keep the problem manageable from a computational point of view) the self-consistent rearrangement of mobile charge within the heterostructure, except for that of the two electrons confined in the cell. In other words, the potential due to the gates is evaluated with the analytical expressions described below and is used as the bare confinement potential for the definition of a many-body Hamiltonian, whose ground state is then evaluated with the configuration-interaction method. The occupancy of the cell is fixed and corresponds to two electrons for all the numerical results we will present. We assume Fermi level pinning at the surface of the semiconductor, so that the electronelectron interaction can be treated by the method of images,${ }^{11}$ without requiring the solution of the Poisson equation.

We have considered two basic gate configurations for the definition of the four quantum dots that make up a cell. The first configuration, represented in Fig. 3, is rather simple and straightforward: the four dots are a consequence of four 


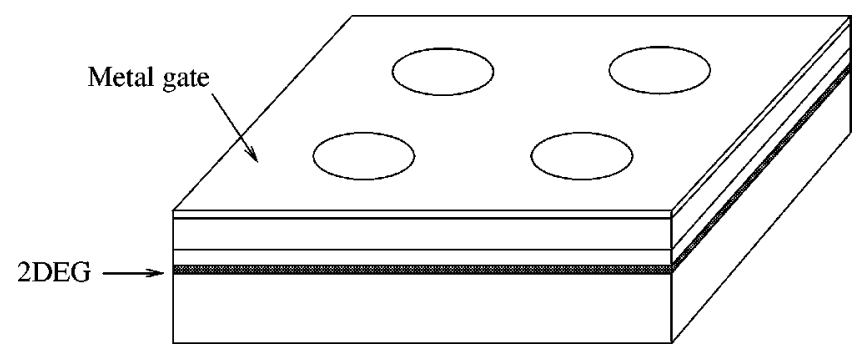

FIG. 3. QCA cell obtained by depleting a two-dimensional electron gas by means of a metallic gate with four holes, which define the four quantum dots.

circular holes in a depleting gate covering the surface of the heterostructure. ${ }^{12}$ The second configuration we have studied is described in Fig. 4 and is more complex: the four dots are defined by a set of seven metal gates which create four minima in the 2D potential at the 2 DEG level.

The contribution to the bare confinement potential from each gate is computed following the method developed in Refs. 9 and 10. The starting point is the well known result that gives the potential inside the semiconductor in terms of its boundary values at the plane of the surface:

$$
V_{g}(x, y, z)=\frac{1}{2 \pi} \int_{S} \frac{|z| V_{g}\left(x^{\prime}, y^{\prime}, 0\right)}{\left(x-x^{\prime}\right)^{2}+\left(y-y^{\prime}\right)^{2}+z^{2}} d x^{\prime} d y^{\prime},
$$

where $z$ is the vertical coordinate, orthogonal to the heterostructure layers, and the integration is performed over the gated surface $S$. Given the applied voltages and the shapes of the gates, the confining potential can be easily computed. For simple shapes one can derive more compact expressions by performing some of the integrals in Eq. (2) analytically. For the cases considered here, we have used Eq. (3.17) of Ref. 9 for gates with circular holes, and the equations in Secs. III and IV of Ref. 10 for polygonal gates.

An example of the results obtained by this procedure is reported in Fig. 5: we show the confinement potential at a depth of $50 \mathrm{~nm}$, produced by four holes with a diameter of

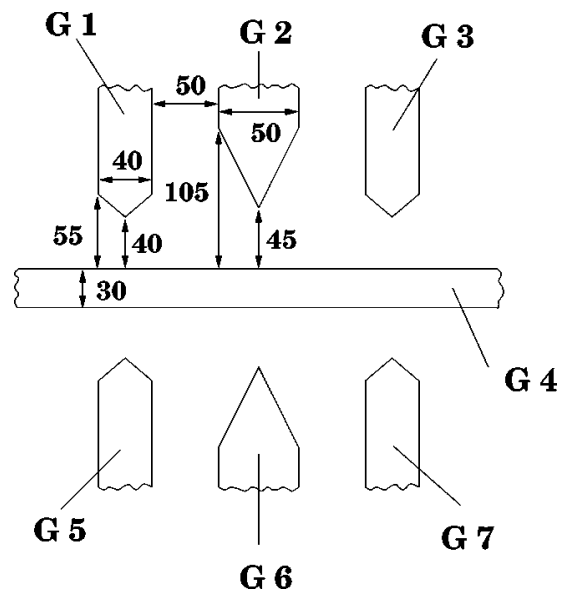

FIG. 4. Gate layout for the definition of four independently adjustable quantum dots; all distances are in nanometers.

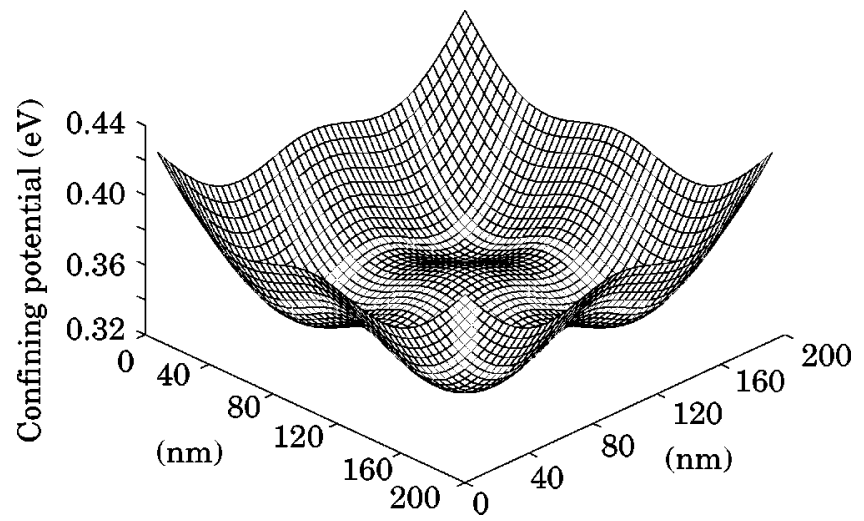

FIG. 5. Confinement potential at a depth of $50 \mathrm{~nm}$ from the surface of the heterostructure, produced by a gate with four holes of $90 \mathrm{~nm}$ diameter and placed at the corners of a $110 \mathrm{~nm}$ square.

$90 \mathrm{~nm}$, with a distance between the centers of $110 \mathrm{~nm}$. The gate voltage has been set at $-0.5 \mathrm{~V}$, in order to obtain interdot barriers of reasonable height.

Following Tougaw et al., ${ }^{8}$ a uniformly distributed positive background charge has been added to each cell. Such a charge does not alter the electrostatic energy splitting between the two cell configurations and plays a role, from the point of view of the cell-to-cell response function, only if the two cells are very close to each other. In this case, it helps prevent alterations of the ground state of the driven cell due to the combined electrostatic repulsion of the two electrons in the driver cell, which would tend to push both electrons into the two dots on the side further from the driver cell itself. In our model, this positive background would not actually be needed to achieve charge neutrality, ${ }^{8}$ which is already ensured by the presence of the gates and of positive charges in the donor layer.

\section{CONFIGURATION-INTERACTION METHOD}

As already mentioned, the solution of a many-electron problem in a potential such as that present in a QCA cell is rather challenging. Approaches that are typically used for the simulation of quantum dots, based on mean-field approximations of the potential seen by each electron and on iterative procedures ${ }^{13-17}$ fail to converge when applied to the fourdot cell. We can understand the reason for this failure considering that convergence of the self-consistent iterative procedures in this class of problems is more and more difficult to achieve as the electrostatic interaction increases,${ }^{18}$ and in the presence of quasidegenerate states. As long as the electrostatic interaction is small compared to the confinement energy, it is just a perturbation of the latter, and iterative self-consistent procedures converge monotonically to the solution. Otherwise, underrelaxation techniques need to be used, but they also often fail when closely spaced states are present, due to symmetries or quasisymmetries in the potential landscape: in this situation the charge will bounce back and forth between quasidegenerate states in consecutive iterations, and convergence is never achieved. Since our calculations are currently performed at zero temperature, in order to compare with experimental results that are typically 
obtained in the tens of millikelvin range, techniques such as the Newton method (which are rather successful in the solution of coupled Schrödinger-Poisson problems at finite temperature) cannot be successfully used due to the sharpness of the Fermi function at low temperatures.

The technique we have implemented is based upon an approach often used in molecular chemistry, ${ }^{7}$ the CI method. It consists of approximating the $N$ electron wave function by a finite linear combination of Slater determinants. Most importantly, the CI method is a one-shot method, i.e., it does not involve an iterative calculation of the wave functions, and, hence, does not suffer from the previously described convergence/oscillation problems.

In order to illustrate the CI method, let us consider an $N$ electron nonrelativistic Hamiltonian with a generic two-body interaction $g\left(\mathbf{r}_{i}, \mathbf{r}_{j}\right)$ :

$$
\begin{aligned}
& \hat{H}=\hat{H}_{1}+\hat{H}_{2}, \\
& \hat{H}_{1}=\sum_{i=1}^{N}\left(-\frac{\hbar^{2}}{2 m} \nabla_{i}^{2}+V\left(\mathbf{r}_{i}\right)\right)=\sum_{i=1}^{N} h\left(\mathbf{r}_{i}\right), \\
& \hat{H}_{2}=\sum_{i<j} g\left(\mathbf{r}_{i}, \mathbf{r}_{j}\right),
\end{aligned}
$$

with

$$
g\left(\mathbf{r}_{i}, \mathbf{r}_{j}\right)=g\left(\mathbf{r}_{j}, \mathbf{r}_{i}\right),
$$

where $\hbar$ is the reduced Planck constant and $m$ is the effective mass of the electron (we consider the case of gallium arsenide, with $m=0.067 m_{0}$, where $m_{0}$ is the free electron mass).

As we are concerned with confined systems, we can consider a numerable complete basis $\left\{\varphi_{i}(\mathbf{q})\right\}$, where $\mathbf{q}=(\mathbf{r}, s)$ includes both spatial and spin coordinates, over which the single-particle wave function can be expanded. In the following, we shall refer to the $\varphi_{i}$ s as spin orbitals. Using this basis, we build all the possible independent Slater determinants:

$$
\Phi_{k}=\frac{1}{\sqrt{N !}}\left|\begin{array}{cccc}
\varphi_{n_{1 k}}\left(\mathbf{q}_{1}\right) & \varphi_{n_{2 k}}\left(\mathbf{q}_{1}\right) & \ldots & \varphi_{n_{N k}}\left(\mathbf{q}_{1}\right) \\
\varphi_{n_{1 k}}\left(\mathbf{q}_{2}\right) & \varphi_{n_{2 k}}\left(\mathbf{q}_{2}\right) & \ldots & \varphi_{n_{N k}}\left(\mathbf{q}_{2}\right) \\
\ldots & \ldots & \ldots & \ldots \\
\varphi_{n_{1 k}}\left(\mathbf{q}_{N}\right) & \varphi_{n_{2 k}}\left(\mathbf{q}_{N}\right) & \ldots & \varphi_{n_{N k}}\left(\mathbf{q}_{N}\right)
\end{array}\right|
$$

where the index $k$ labels the Slater determinants and the integer $n_{j k}$ specifies which spin orbital appears in the $j$ th column of the $k$ th Slater determinant.

The infinite set $\left\{\Phi_{k}\right\}$ is a complete orthonormal basis for the $N$ electron eigenfunctions of the Hamiltonian in Eq. (3) ${ }^{19}$ the $i$ th eigenfunctions $\Psi_{i}$ can therefore be written as

$$
\Psi_{i}=\sum_{k=1}^{\infty} c_{i k} \Phi_{k}
$$

To find the eigenfunctions of $\hat{H}$, we must solve the secular equation

$$
\mathcal{H} \mathbf{c}_{i}=E_{i} \mathbf{c}_{i},
$$

where the infinite-dimensional "Hamiltonian matrix" is

$$
\mathcal{H}_{k k^{\prime}}=\left\langle\Phi_{k}|\hat{H}| \Phi_{k^{\prime}}\right\rangle=\int \Phi_{k}^{*}\left(\hat{H}_{1}+\hat{H}_{2}\right) \Phi_{k^{\prime}} \prod_{i=1}^{N} d \mathbf{q}_{i},
$$

$E_{i}$ is the $i$ th eigenvalue of $\mathcal{H}$, the vector $\mathbf{c}_{i}$ is made up of the coefficients $c_{i k}$ and $\int d \mathbf{q}_{i}$ stands for integration over the $i$ th spatial coordinate and summation over spin orientations.

In practice, this approach cannot be implemented "exactly" (i.e., choosing a complete, infinite set of orthonormal spin orbitals). In our numerical work, we consider a finite set of $M$ spin orbitals $\left\{\varphi_{j}(\mathbf{q})\right\}$, with $j=1 \cdots M$.

Given $N$ electrons and $M$ spin orbitals (with $M \geqslant N$ ), it is possible to build $\mathcal{N}_{\mathrm{SD}}$ different Slater determinants, where

$$
\mathcal{N}_{\mathrm{SD}}=\left(\begin{array}{c}
M \\
N
\end{array}\right)
$$

With this choice, the secular equation (8) becomes an $\mathcal{N}_{\mathrm{SD}}$ $\times \mathcal{N}_{\text {SD }}$ Hermitian eigenvalue problem.

The number of nonzero matrix elements is less than $\left(\mathcal{N}_{\mathrm{SD}}\right)^{2}$, because all the matrix elements between determinants differing by more than two spin orbitals do vanish, as a consequence of the selection rules (Slater's rules ${ }^{7}$ ) presented in Appendix A, where the general expressions for the elements of $\mathcal{H}$ are also provided.

The total number $\mathcal{M}_{\mathrm{NZ}}$ of nonzero matrix elements is given by the following expression:

$$
\begin{aligned}
\mathcal{M}_{\mathrm{NZ}}= & \left(\begin{array}{c}
M \\
N
\end{array}\right) \times\left[1+\left(\begin{array}{c}
M-N \\
1
\end{array}\right)\left(\begin{array}{c}
N \\
1
\end{array}\right)+\left(\begin{array}{c}
M-N \\
2
\end{array}\right)\left(\begin{array}{c}
N \\
2
\end{array}\right)\right] \\
= & \left(\begin{array}{c}
M \\
N
\end{array}\right) \times(1+(M-N) N \\
& \left.+\frac{(M-N)(M-N-1) N(N-1)}{4}\right) .
\end{aligned}
$$

Once the eigenvectors of Eq. (8) have been obtained, the $N$ electron wave function $\Psi_{i}$ can be computed from Eq. (7), and the corresponding electron density is simply given by:

$$
\rho_{i}\left(\mathbf{r}_{1}\right)=N \sum_{s_{1}} \int\left|\Psi_{i}\left(\mathbf{r}_{1}, s_{1}, \mathbf{q}_{2}, \cdots, \mathbf{q}_{N}\right)\right|^{2} d \mathbf{q}_{2} \cdots d \mathbf{q}_{N},
$$

where $s_{1}$ represents the spin orientation coordinate.

Since we are going to present numerical results for a cell occupancy of two electrons, we now focus our attention on the two-electron case. The Hamiltonian for the structure under study can be written:

$$
\begin{aligned}
\hat{H}= & -\frac{\hbar^{2}}{2 m} \nabla_{1}^{2}-\frac{\hbar^{2}}{2 m} \nabla_{2}^{2}+V_{\text {con }}\left(\mathbf{r}_{1}\right)+V_{\text {con }}\left(\mathbf{r}_{2}\right)+V_{\text {driv }}\left(\mathbf{r}_{1}\right) \\
& +V_{\text {driv }}\left(\mathbf{r}_{2}\right)+g\left(\mathbf{r}_{1}, \mathbf{r}_{2}\right)
\end{aligned}
$$

where $V_{\text {con }}$ is the confinement potential computed as described in Sec. II, $V_{\text {driv }}$ is the Coulomb potential due to the charge distribution in the neighboring driver cell, and $g\left(\mathbf{r}_{1}, \mathbf{r}_{2}\right)$ is the two-body interaction. The two-body interaction includes the effects of image charges and is given by: 


$$
\begin{aligned}
g\left(\mathbf{r}_{1}, \mathbf{r}_{2}\right)= & \frac{1}{4 \pi \epsilon} \frac{e^{2}}{\left|\mathbf{r}_{1}-\mathbf{r}_{2}\right|}-\frac{1}{4 \pi \epsilon} \frac{e^{2}}{\sqrt{\left|\mathbf{r}_{1}-\mathbf{r}_{2}\right|^{2}+(2 z)^{2}}} \\
& -\frac{1}{4 \pi \epsilon} \frac{e^{2}}{2 z}
\end{aligned}
$$

where $\epsilon=\epsilon_{r} \epsilon_{0}$ (where $\epsilon_{r}$ and $\epsilon_{0}$ are the relative permittivity of gallium arsenide and the vacuum permittivity, respectively) and $e$ is the electron charge. This expression has been obtained by taking one half of the electrostatic energy of the system made up of the electrons and their image counterparts, since the energy stored in the image space is purely fictitious. The last term of Eq. (14), due to the interaction of each electron with its own image, yields a constant shift of the energy spectrum.

To apply the CI method to the system described by the Hamiltonian in Eq. (13), we start by choosing a set of $n$ wave functions $\left\{\psi_{i}(\mathbf{r})\right\}$. We shall refer to the $\left\{\psi_{i}(\mathbf{r})\right\}$ s as orbitals, to distinguish them from the spin orbitals $\left\{\varphi_{i}(\mathbf{q})\right\}$. By combining each of the $\psi_{i}$ s with one of the two possible spin eigenstates corresponding to the two spin orientations along the $z$ axis, we obtain the set $\left\{\varphi_{i}(\mathbf{q})\right\}$ of $M=2 n$ spin orbitals.

With $M=2 n$ spin orbitals and two electrons, we can construct $n(2 n-1)$ independent Slater determinants [see Eq. (10)]. In the expansion of Eq. (7), instead, we take into account only $n^{2}$ Slater determinants, i.e., those composed of spin orbitals with opposite spins that correspond to states with zero total spin component $S_{z}$ along the $z$ axis. These states are, in general, linear combinations of the singlet state $\left|S=0, S_{z}=0\right\rangle$ and of the triplet state $\left|S=1, S_{z}=0\right\rangle$. Since we are dealing with a spin-independent Hamiltonian, the triplet states $\left|S=1, S_{z}=0\right\rangle,\left|S=1, S_{z}= \pm 1\right\rangle$ are degenerate in energy; in addition, the corresponding wave functions have the same spatial part. Therefore, no information about the energy eigenstates of the system is lost, if only one of the triplet states is used to expand the wave function of Eq. (7).

With the above mentioned restriction on the number of Slater determinants, the matrix $\mathcal{H}$ is an $n^{2} \times n^{2}$ Hermitian matrix. We can choose the orbitals $\psi_{i}$ to be real, thus making the matrix $\mathcal{H}$ real and symmetric. Finally we note that $\mathcal{H}$ is a full matrix since the $\Phi_{k} \mathrm{~s}$, being $2 \times 2$ determinants, cannot differ by more than two spin orbitals.

The choice of the set of orbitals $\left\{\psi_{i}\right\}$ is of crucial importance, since the number $n$ of orbitals required to get a satisfactory approximation of the ground state energy and the corresponding wave function depends on it. We have used the single-electron eigenstates for an isolated cell as orbitals and we have found that with this basis 12 orbitals (i.e., 24 spin orbitals) are sufficient to get good accuracy in the results for cell sizes around $200 \mathrm{~nm}$. In order to check the validity of the approximation, we have also performed calculations using 24 orbitals, finding that the results are practically identical to those obtained with the smaller basis. For larger cell sizes, a larger number of orbitals would be necessary, because the electrostatic interaction would grow in importance compared to the confinement energy. Therefore, the ground state wave function would deviate further from a single Slater determinant built with the single-electron orbit- als, and would thus need to be expanded on a basis of Slater determinants built from a larger set of single-electron wave functions.

Let us briefly comment on the relationship of the solution obtained with this method to those obtained with other commonly used approaches for the self-consistent solution of the electronic structure of quantum dots. In the Hartree method $^{17}$ and in the local density functional approximation (LDA), ${ }^{16}$ the exchange term is ignored or treated in an approximate fashion, while the wave functions are obtained at each iteration as eigenfunctions of a modified Hamiltonian. When convergence is reached, the obtained wave functions are the set of one-electron wave functions minimizing the expectation value of the approximate Hamiltonian.

In the case of the Hartree-Fock method ${ }^{20}$ the manyelectron wave function is represented by a single Slater determinant, and when self-consistency is attained, the resulting Slater determinant minimizes the expectation value of the Hamiltonian, as in the case of the Hartree and LDA methods, but with a properly antisymmetrized wave function.

With the CI method, the wave function is expanded over a basis of Slater determinants, which guarantees proper antisymmetrization. The basis functions are fixed and the unknowns are the coefficients of the expansion. If the basis were complete (comprising an infinite number of spin orbitals), the solution would be exact and corresponding to that obtainable by diagonalizing the many-body Hamiltonian. In order to make the problem computationally feasible, we must limit the number of basis functions introducing, as a result, some approximation. The difference between the solutions obtained with the application of the Hartree-Fock method and of the CI method can thus be summarized as follows: with the former we get a single, optimized Slater determinant, while with the latter we obtain an expansion of the solution over a finite basis of Slater determinants which have been chosen a priori. In the presence of strong electronelectron correlation, the wave function obtained by the CI method with the inclusion of a reasonable number of basis functions is expected to be much closer to the exact solution than the optimized Slater determinant resulting from the Hartree-Fock method.

\section{RESULTS AND DISCUSSION}

\section{A. Single gate configuration with four circular holes}

We start the presentation of the numerical results with the cell-to-cell response function obtained for a cell defined by a gate with four circular holes, for a choice of 4,12 , and 24 basis orbitals (Fig. 6). Each cell is defined by holes with a diameter of $90 \mathrm{~nm}$, placed at the corners of a square, with a distance of $110 \mathrm{~nm}$ between the hole centers. The applied gate bias is $-0.5 \mathrm{~V}$ and the separation between the centers of the two cells is $400 \mathrm{~nm}$. We found that there is no significant difference between the results obtained with the three choices of basis elements. In some other cases we have noticed a small difference between the response function calculated with four basis orbitals and those for 12 and 24 or- 


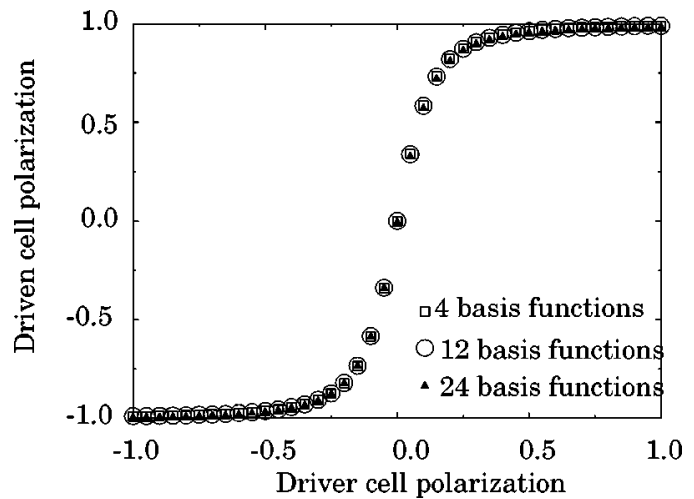

FIG. 6. Cell-to-cell response function obtained for a cell defined by a gate, kept at $-0.5 \mathrm{~V}$, with four $90 \mathrm{~nm}$ holes placed at the corners of a $110 \mathrm{~nm}$ square. The depth of the 2DEG is $50 \mathrm{~nm}$ and the separation between the centers of the driven and the driver cells is $400 \mathrm{~nm}$. The different symbols indicate different numbers of basis functions.

bitals, which were instead found to be practically identical. All the results presented in the following have therefore been obtained with a basis of 12 orbitals.

An example of the electron density, computed for full polarization, is reported in Fig. 7. The distance of the 2DEG from the surface of the heterostructure plays an important role and significantly affects the cell-to-cell response function: the closer it lies to the surface, the higher (for a given value of the bias voltage) the potential barriers separating the dots in a cell are and as a result, the steeper and more abrupt the response function is. This is the prevailing effect, even though it is partially compensated for by the screening action of the surface, which reduces the cell-to-cell interaction and increases with decreasing distance.

In Fig. 8(a) we show a polarization curve which has been obtained for the same dot configuration as that in Fig. 6, but for different values of the 2DEG depth, from 45 to 55 $\mathrm{nm}$. As expected, the polarization curve becomes smoother for increasing depth of the 2DEG. The screening effect from the gates can be appreciated by comparison with the results obtained neglecting the contribution due to the images. This is seen in Fig. 8(b), where we report the cell-to-cell response function for the previously considered gate geometry and for a 2 DEG depth of $50 \mathrm{~nm}$, computed with and without images.

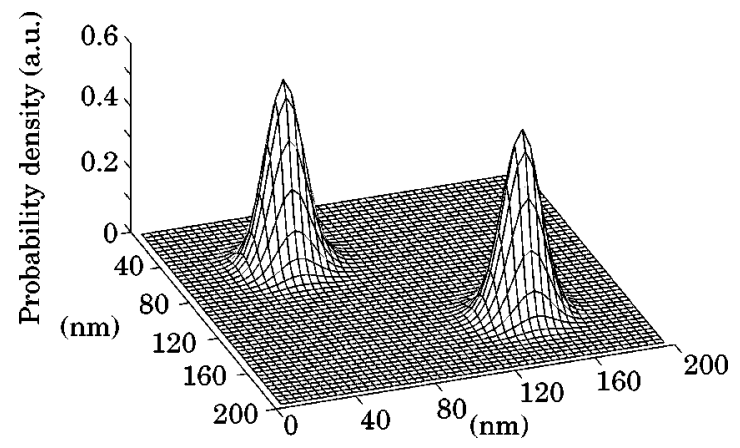

FIG. 7. Electron density (in arbitrary units) for a completely polarized cell defined by a gate, kept at $-0.5 \mathrm{~V}$, with four $90 \mathrm{~nm}$ holes placed at the corners of a $110 \mathrm{~nm}$ square. The depth of the $2 \mathrm{DEG}$ is $50 \mathrm{~nm}$.
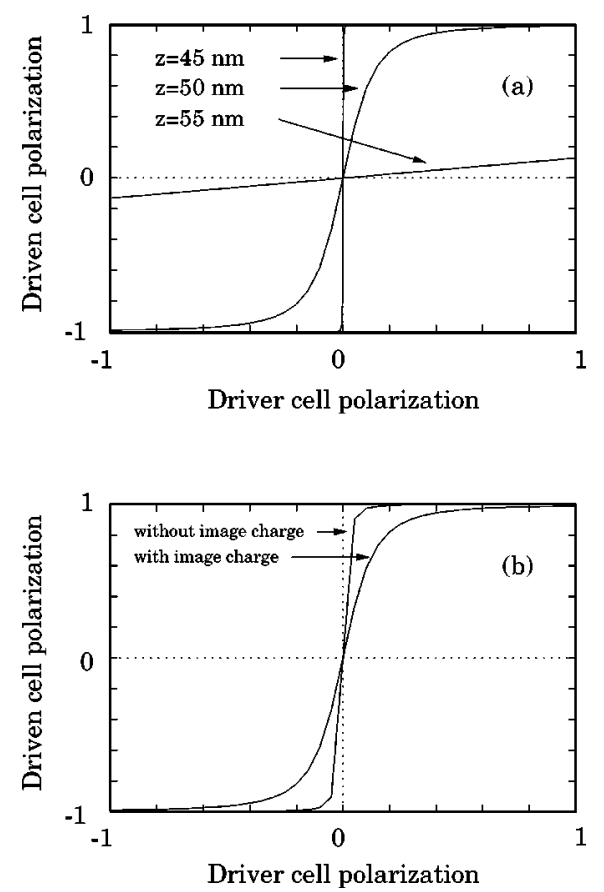

FIG. 8. Cell-to-cell response functions obtained for a cell defined by a gate, kept at $-0.5 \mathrm{~V}$, with four $90 \mathrm{~nm}$ holes placed at the corners of a $110 \mathrm{~nm}$ square. The separation between the centers of the two cells is $400 \mathrm{~nm}$. (a) Comparison between the response functions for different values of the 2DEG depth. (b) Comparison between the response functions obtained with and without the contribution of the image charges.

The image effects decrease the sharpness of the response function because the dipole moment of the driver cell is screened by its images.

As stated in Sec. I, we have applied our CI technique to assess the sensitivity of a two-cell structure to fabrication tolerances. First we considered giving one of the holes in the gate a diameter slightly different from the others: this leads to a variation in the potential landscape defining the cell and, in particular, to a modification of the confinement energy associated with that dot. If we reduce the diameter of one of the holes, the confinement energy for the dot underneath will rise by a certain amount $\delta E$. If $\delta E$ is larger than the electrostatic splitting $\Delta E_{C}$ between the two cell configurations, the cell will be stuck in a state with the smaller dot empty. For $\delta E<\Delta E_{C}$, the cell will still be operational, but the cell-tocell response function will be shifted by an amount which depends on the ratio of $\delta E$ to $\Delta E_{C}$. The strong nonlinearity of the response function helps to restore the correct polarization value along a chain of cells, as long as the shift still allows full polarization of the driven cell for full polarization of the driver cell.

The tolerance on the hole diameter, admissible before unrecoverable disruption of the operation of two coupled cells occurs, is unfortunately very small: from our calculations it is about one part in 10000 for the dot sizes considered so far. Since the tolerance is determined by the interplay between the electrostatic splitting energy (which has an inverse linear dependence on the size) and the perturbation of the confinement energy (which has an inverse quadratic dependence on the size) it will be even tighter for smaller cells. 

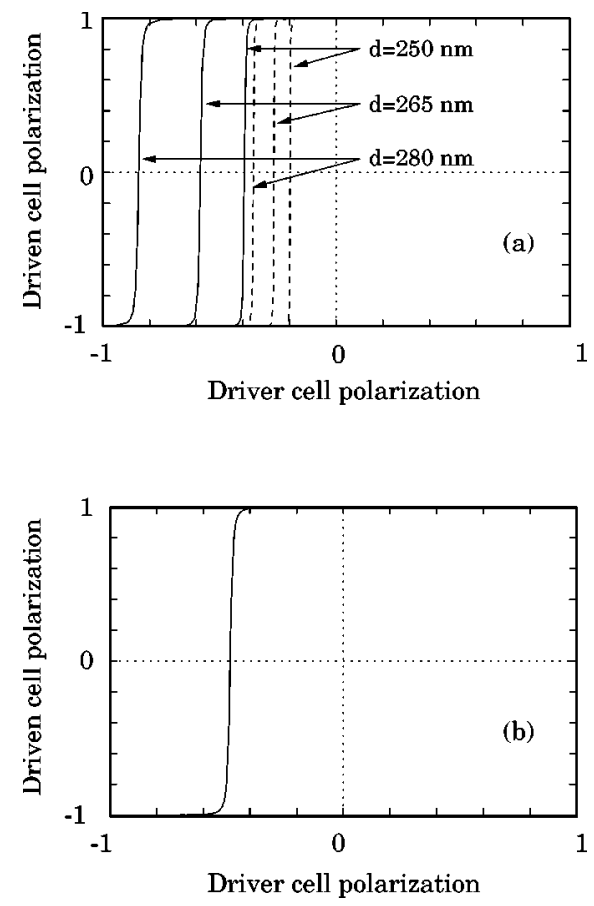

FIG. 9. Cell-to-cell response functions obtained for a cell defined by a gate, kept at $-0.5 \mathrm{~V}$, with four holes placed at the corners of a $110 \mathrm{~nm}$ square. (a) The bottom left hole has a diameter of $89.991 \mathrm{~nm}$ and the other three holes of $90 \mathrm{~nm}$; the depth of the 2DEG is $50 \mathrm{~nm}$. Results for three different values of the separation between the cell centers are reported, including the effect of image charges (solid lines) or neglecting it (dashed lines). (b) The four holes have a diameter of $90 \mathrm{~nm}$, but the bottom left hole has been moved down and to the left by $0.0275 \mathrm{~nm}$.

In Fig. 9(a) we report the cell-to-cell response function for a gate configuration as previously described, with the diameter of one of the dots reduced to 0.9999 times the nominal value. The depth of the $2 \mathrm{DEG}$ is assumed to be 50 $\mathrm{nm}$ and the three solid curves refer to different separations between cell centers. It is clear that for a separation between the centers greater than $280 \mathrm{~nm}$, an error of one part in 10000 will be sufficient to unrecoverably disrupt QCA operation. For purposes of comparison, we have also reported the results (dashed curves) obtained neglecting the contribution from the image charges (i.e., the screening due to the gates and to the assumed Fermi level pinning at the semiconductor-air interface).

We have also investigated the sensitivity to errors in the position of the gate holes. With a hard-wall model (such as in Ref. 8) a small shift in the position of one hole would not have a disrupting effect, since it would cause only a proportionately small variation of the electrostatic splitting and would not affect the confinement energy in any way. With a realistic model (as in Ref. 8) the situation is quite different: the confinement potential for each dot is determined not only by the corresponding hole, but also by the other holes belonging to the same cell. This means that by shifting a hole away from its nominal position, the potential landscape defining the dot underneath will be distorted, and the confinement energy will change. As a consequence, cell operation will be disrupted for unexpectedly small errors in hole positioning. In Fig. 9(b) we report the cell-to-cell response function obtained for a $0.0275 \mathrm{~nm}$ shift down and to the left of

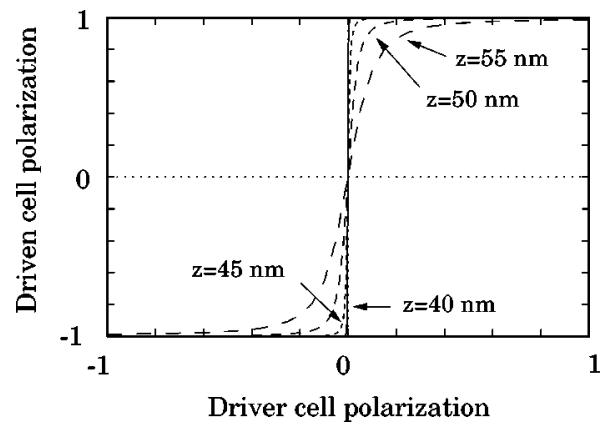

FIG. 10. Cell-to-cell response functions obtained for a cell defined with the seven-gate layout and a distance between cell centers of $280 \mathrm{~nm}$. The gates are kept at $-1.8 \mathrm{~V}$, except for gates 2 and 6 , which are kept at $-1.6 \mathrm{~V}$. Results for a depth of the 2DEG varying between 40 and $55 \mathrm{~nm}$ have been reported.

the bottom left dot of the driven cell. For the rest, the cell configuration is unchanged: a dot diameter of $90 \mathrm{~nm}$, a dotdot separation within a cell of $110 \mathrm{~nm}$ and a distance between cell centers of $280 \mathrm{~nm}$. Such a small displacement is sufficient to significantly shift the response function; a 0.05 $\mathrm{nm}$ shift leads already to the breakdown of cell operation.

\section{B. Multiple independent gates with adjustable voltages}

From the results shown above, it is clear that a simple hole-array implementation of QCA cells leads to unrealizable requirements on fabrication tolerances. This is the reason why we have also investigated alternative gate layouts such as that sketched in Fig. 4. If dot confinement is obtained via multiple independent gates, it is indeed possible to compensate for geometrical tolerances by adjusting the gate voltages.

In Fig. 10 we show the cell-to-cell response function for a cell defined with bias voltages of $-1.8 \mathrm{~V}$ applied to all gates, except for gates 2 and 6 , which are fixed at $-1.6 \mathrm{~V}$; these bias values have been chosen within a reasonable voltage range so as to get four clearly confined dots. The separation between the centers of the driver and the driven cells is $280 \mathrm{~nm}$. Also in this case, different depths for the 2DEG have been considered: from 40 to $55 \mathrm{~nm}$.

A cross section of the confinement potential, cut across the two upper dots, is shown in Fig. 11 for a 2DEG at $35 \mathrm{~nm}$

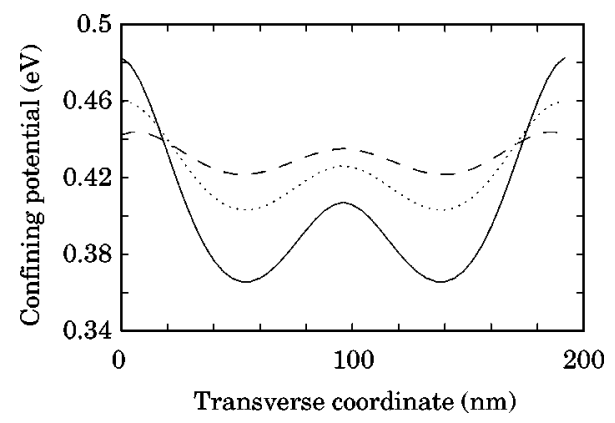

FIG. 11. Cross section of the confining potential obtained with the sevengate layout, cut across the two upper dots, at a depth from the surface of 35 $\mathrm{nm}$ (solid line), $45 \mathrm{~nm}$ (dotted line), and $55 \mathrm{~nm}$ (dashed line). 

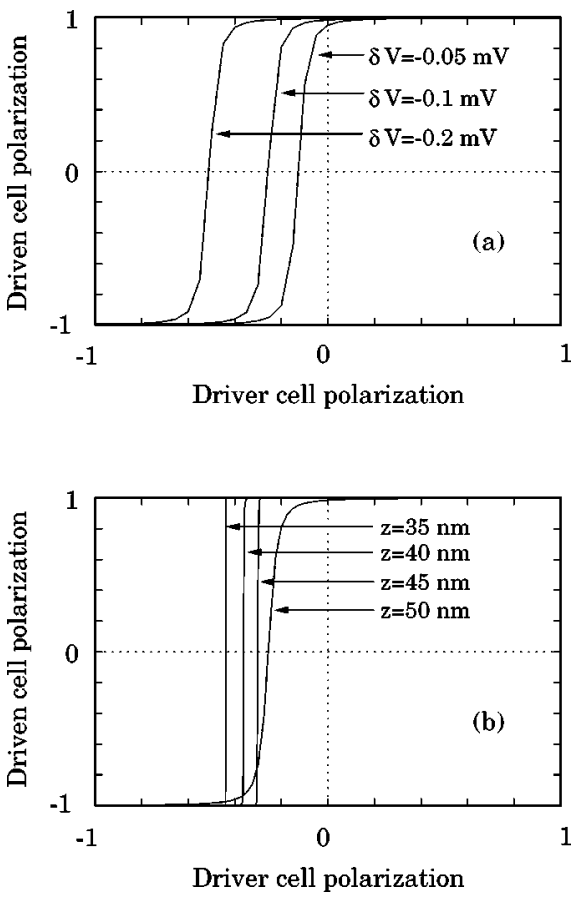

FIG. 12. Cell-to-cell response functions obtained for a cell defined with the seven-gate layout and for a distance between cell centers of $280 \mathrm{~nm}$. The gates are kept at $-1.8 \mathrm{~V}$, except for gates 2 and 6 , which are kept at $-1.6 \mathrm{~V}$, and gate 3 , which is kept at $-1.8 \mathrm{~V}+\delta V$. (a) The depth of the 2DEG is $50 \mathrm{~nm}$ and the results for three different values of $\delta V$ have been reported. (b) The value of $\delta V$ is $-0.1 \mathrm{mV}$ and results for different depths of the $2 \mathrm{DEG}$ are shown.

(solid line), $45 \mathrm{~nm}$ (dotted line) and $55 \mathrm{~nm}$ (dashed line): the height of the barriers is relatively low, but their width guarantees a low enough transparency for strong localization of the electrons and thereby correct operation of the cell.

We have also investigated the dependence of the cell-tocell response function on electrical asymmetries, for the case of a 2DEG $50 \mathrm{~nm}$ depth and a distance of $280 \mathrm{~nm}$ between the centers of the driver and the driven cell. For this purpose, the voltage applied to gate 3 has been made slightly more negative by an amount $\delta V$. The results for $\delta V=-0.05$, $-0.1,-0.2 \mathrm{mV}$ are shown in Fig. 12(a). The most visible effect is a shift in the cell-to-cell response function, which is somewhat proportional to the variation in the applied voltage. This does not disrupt the operation of a QCA chain, as long as full polarization of one cell can produce full polarization of the neighboring cell. Therefore, for this particular cell, we expect a maximum tolerance on the gate voltages of about $0.4 \mathrm{mV}$. This may seem difficult to achieve at first sight, but it is important to keep in mind that it is a shift between gate biases: larger variations in the overall average value of the gate voltages are allowed, as long as they do not alter cell occupancy.

As previously mentioned, the screening effect due to the gates and to the charge at the semiconductor-air interface decreases the strength of the electrostatic interaction and, therefore, the energy splitting between the two possible cell polarizations. Hence, the effects of asymmetry decrease with increasing depth of the 2DEG, as the image effects are reduced. This phenomenon is clearly visible in Fig. 12(b),
TABLE I. Values of the bias voltages to be applied to the gates defining the geometrically asymmetric cell shown in Fig. 4, in order to symmetrize it.

\begin{tabular}{cc}
\hline \hline Gate & Bias voltage $(\mathrm{V})$ \\
\hline$V_{1}$ & -1.665577 \\
$V_{2}$ & -1.728683 \\
$V_{3}$ & -1.845800 \\
$V_{4}$ & -1.800000 \\
$V_{5}$ & -1.807502 \\
$V_{6}$ & -1.592665 \\
$V_{7}$ & -1.793715 \\
\hline \hline
\end{tabular}

where the cell-to-cell response function is plotted for a voltage shift of $-0.1 \mathrm{mV}$ on gate 3 and various values of the 2DEG depth ranging from 35 to $50 \mathrm{~nm}$. The shift in the response function decreases as the depth of the 2DEG increases, and the effect of the image term becomes less important.

Fabrication tolerances would also disrupt the operation of this type of cell, but they can be compensated for by fine adjustments to the bias voltages applied at the gates. As an example, we consider a cell with gate 3 shifted by $5 \mathrm{~nm}$ to the right. An iterative procedure was developed for computing the new bias voltages that will restore the symmetry of the structure. For a perfectly symmetric structure there is a fourfold quasidegeneracy of the one-electron states, corresponding to the fourfold symmetry of the cell. When symmetry is disrupted, this quasidegeneracy is lifted, and the first four eigenvalues differ from each other by an amount which is no longer negligible. Our strategy is to evaluate the difference between the first and the fourth eigenvalue, and then adjust each gate voltage in such a way as to minimize this difference. We vary one gate voltage at a time, from gate 1 to gate 7 (with the exception of gate 4, which does not affect cell symmetry), and then repeat the cycle, starting again from gate 1, until the splitting between the first and the fourth eigenvalue is smaller than an assigned threshold. In order to avoid getting stuck in local minima, it is convenient to perform the whole minimization procedure several times, for values of the displacement of gate 3 increasing with a geometric progression from 0.01 to $5 \mathrm{~nm}$. Finally, small adjustments are made manually, until a symmetric cell-to-cell response function is obtained. The gate voltages needed to symmetrize the cell are listed in Table I, while the cell response function for the symmetrized cell is shown in Fig. 13. This demonstrates that a $10 \%$ error in the position of one of the gates can be fully compensated. State of the art fabrication techniques allow geometrical tolerances of this order of magnitude or smaller, thus such a cell is actually manufacturable, although not useful for large scale applications where it would be impossible to tune each cell separately. By acting on the gate voltages it is also possible to compensate for the presence of randomly distributed stray charges, which would also disrupt QCA operation. We notice that the cellto-cell response function of Fig. 13 is steeper than that for a geometrically symmetric cell with analogous parameters (see Fig. 10). This is a consequence of a slight variation in the barrier heights and widths (due to the different applied voltages) and of the exponential dependence of the tunneling 


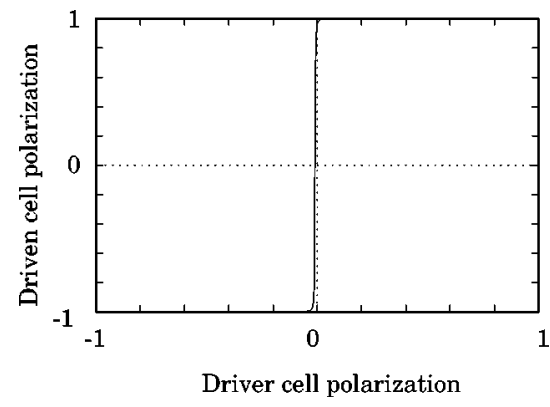

FIG. 13. Cell-to-cell response functions obtained for a cell defined with the seven-gate layout, a distance between cell centers of $280 \mathrm{~nm}$, and a 2DEG depth of $50 \mathrm{~nm}$. Gate 3 has been shifted to the right by $5 \mathrm{~nm}$, and the bias voltages are those listed in Table I, chosen to restore cell symmetry.

coefficients between the dots on such barrier characteristics. A chain of such cells can be fabricated by repeating this same gate layout in the horizontal direction. However lateral branching, needed for the implementation of logic gates, ${ }^{1}$ is not allowed due to the lateral extension of the leads required for feeding the bias voltages. From this point of view, a more promising implementation would be that suggested by Chen and Porod, ${ }^{12}$ with central enhancement gates in each dot: by adjusting the voltage of such a central gate it would be possible to correct asymmetries, while keeping the possibility of lateral branching. This implementation, however, poses serious fabrication problems because of the difficulties involved in separately contacting all of the central gates.

We emphasize that the compensation procedure described above is not proposed as a practical method, but rather our aim has been to demonstrate that compensation is possible in principle. In an actual experiment a different procedure must be followed. One can, for example, look at the currents through the two upper (lower) dots by applying a very small voltage between the upper left (lower left) and the upper right (lower right) outer portions of the 2DEG, current maxima will be detected when the chemical potential in both dots lines up with that in the 2DEG. Gate voltages should be iteratively adjusted in such a way as to obtain a maximum in the currents through both pairs of dots. Once this is achieved, the system will be symmetrized, and the bias voltages between the different portions of the outer 2DEG can be removed. As a result of the symmetrization method we propose, the total occupancy of the cell is not guaranteed to be of just two electrons, but cell functionality will be preserved. A detailed simulation of such a procedure is rather complex and is beyond the scope of the present article; it will be presented elsewhere.

\section{CONCLUSIONS}

We have shown that the CI method allows us to solve the many-electron Schrödinger equation for a QCA cell made up of four coupled dots. This represents a definite advance over the state of the art in the simulation of multiple quantum dot systems, it is possible to include realistic confinement potentials without resorting to some type of meanfield approximation (local density approximation, HartreeFock, Hartree-Fock-Roothaan) which fail to converge when single-electron states are strongly degenerate and the electrostatic interaction is comparable to the confinement energy. A Hubbard-like approach to QCA cells is also feasible and has been shown to provide a qualitative understanding of the underlying physics, ${ }^{8}$ but requires a set of phenomenological parameters such as of the on-site electrostatic interaction, the dot confinement energy and the tunneling energy, which cannot be easily obtained from the geometrical structure and from experiments. With the CI method it is sufficient to determine the confinement potential from the layer structure and the gate layout. This is an important advantage that makes simulations based on the CI method a reliable and effective design tool.

A disadvantage of the $\mathrm{CI}$ approach lies in the large computational resources which are required, since the number of Slater determinants to be considered exhibits a combinatorial increase with the number of electrons in the system. In this paper, we have presented results for two electrons. (A QCA cell with up to six electrons is currently being investigated.) Calculations for a larger number of electrons would require prohibitive memory sizes (well above 1 Gbyte) or extremely long computation times.

We have focused our investigation on the sensitivity to fabrication tolerances for two coupled QCA cells. Our results demonstrate that the implementation of a simple hole-array approach is not feasible because it would require a precision in the diameter of each hole that is well beyond the present state of the art in electron-beam lithography.

We have proposed an alternative cell layout, based on seven gates, whose bias voltages can be independently adjusted, this approach is within the capabilities of current fabrication technologies, since geometrical errors in the gate positions can be corrected by means of appropriate voltage variations. Admissible voltage tolerances are rather small, but achievable using resistive voltage dividers cooled down together with the sample. The approach we propose should allow an experimental demonstration of the QCA principle, from a single cell up to a chain of cells, but it is not suitable for the realization of logic gates due to the impossibility of lateral branching, which is prevented by the leads reaching each gate. It is also impractical for large-scale integration due to the need for individual adjustment of each single cell. Viable logic circuits will require drastically different solutions and new architectural concepts.

\section{ACKNOWLEDGMENTS}

The authors thank Professor C. Guidotti for her useful suggestions on the CI method and Professor D. W. L. Sprung for valuable discussion. This work has been supported by the ESPRIT Project No. 23362 QUADRANT (quantum devices for advanced nanoelectronic technology).

\section{APPENDIX A}

The problem of computing the matrix elements of the Hamiltonian in Eq. (3) between two Slater determinants is well known. ${ }^{19}$ For the diagonal elements one finds: 


$$
\begin{aligned}
\left\langle\Phi_{k}|\hat{H}| \Phi_{k}\right\rangle= & \sum_{i}\left\langle\varphi_{n_{i k}}|h| \varphi_{n_{i k}}\right\rangle \\
& +\frac{1}{2} \sum_{i j}\left(\left\langle\varphi_{n_{i k}} \varphi_{n_{j k}}|g| \varphi_{n_{i k}} \varphi_{n_{j k}}\right\rangle\right. \\
& \left.-\left\langle\varphi_{n_{i k}} \varphi_{n_{j k}}|g| \varphi_{n_{j k}} \varphi_{n_{i k}}\right\rangle\right),
\end{aligned}
$$

where in general

$$
\begin{aligned}
\left\langle\varphi_{i} \varphi_{j}|g| \varphi_{l} \varphi_{m}\right\rangle= & \int d q_{1} d q_{2} \varphi_{i}^{*}\left(q_{1}\right) \varphi_{j}^{*}\left(q_{2}\right) g\left(\mathbf{r}_{1}, \mathbf{r}_{2}\right) \\
& \times \varphi_{l}\left(q_{1}\right) \varphi_{m}\left(q_{2}\right) .
\end{aligned}
$$

As far as the computation of the off-diagonal matrix elements of the Hamiltonian of Eq. (3) between two different Slater determinants $\left(\Phi_{k}, \Phi_{k^{\prime}}\right)$ is concerned, there are some "selection rules" (Slater's rules ${ }^{7}$ ) which state that there are only two possible cases in which $\left\langle\Phi_{k}|\hat{H}| \Phi_{k^{\prime}}\right\rangle$ is not vanishing, i.e., when $\Phi_{k}, \Phi_{k^{\prime}}$ either differ by one single spin orbital or by two:

(1) one spin-orbital difference $\left(\varphi_{n_{i k}} \neq \varphi_{n_{i k^{\prime}}}\right)$

$$
\begin{aligned}
\left\langle\Phi_{k}|\hat{H}| \Phi_{k^{\prime}}\right\rangle= & \left\langle\varphi_{n_{i k}}|h| \varphi_{n_{i k^{\prime}}}\right\rangle+\sum_{j \neq i}\left(\left\langle\varphi_{n_{i k}} \varphi_{n_{j k}}|g| \varphi_{n_{i k^{\prime}}} \varphi_{n_{j k}}\right\rangle\right. \\
& \left.-\left\langle\varphi_{n_{i k}} \varphi_{n_{j k}}|g| \varphi_{n_{j k}} \varphi_{n_{i k^{\prime}}}\right\rangle\right),
\end{aligned}
$$

(1) two spin-orbital difference $\left(\varphi_{n_{i k}} \neq \varphi_{n_{i k^{\prime}}}\right.$ and $\left.\varphi_{n_{j k}} \neq \varphi_{n_{j k^{\prime}}}\right)$

$$
\left\langle\Phi_{k}|\hat{H}| \Phi_{k^{\prime}}\right\rangle=\left\langle\varphi_{n_{i k}} \varphi_{n_{j k}}|g| \varphi_{n_{i k^{\prime}}} \varphi_{n_{j k^{\prime}}}\right\rangle-\left\langle\varphi_{n_{i k}} \varphi_{n_{j k}}|g| \varphi_{n_{j k^{\prime}}} \varphi_{n_{i k^{\prime}}}\right\rangle .
$$

The expressions in Eqs. (A3) and (A4) refer to the case in which the spin orbitals that are common to both Slater determinants occur in the same columns. If this is not the case, it is possible to perform a permutation of the columns of one determinant so that the above condition is satisfied; the permutation has the effect of changing the sign of the matrix element if it is an odd order permutation.

Finally, it is worth noting that Eqs. (A1), (A3), and (A4) are valid only if the orthonormality condition on the spin orbitals is satisfied.

${ }^{1}$ P. D. Tougaw and C. S. Lent, J. Appl. Phys. 75, 1818 (1994).

${ }^{2}$ C. S. Lent, P. D. Tougaw, and W. Porod, Appl. Phys. Lett. 62, 714 (1993).

${ }^{3}$ P. Bakshi, D. A. Broido, and K. Kempa, J. Appl. Phys. 70, 5150 (1991).

${ }^{4}$ S. Bandyopadhyay, B. Das, and A. E. Miller, Nanotechnology 5, 113 (1994).

${ }^{5}$ C. S. Lent and P. D. Tougaw, J. Appl. Phys. 74, 6227 (1993).

${ }^{6} \mathrm{R}$. Landauer, IEEE Trans. Electron Devices 43, 1637 (1996).

${ }^{7}$ R. McWeeny, Methods of Molecular Quantum Mechanics (Academic, London, 1989), and references therein.

${ }^{8}$ P. D. Tougaw, C. S. Lent, and W. Porod, J. Appl. Phys. 74, 3558 (1993).

${ }^{9}$ J. H. Davies, Semicond. Sci. Technol. 3, 995 (1988).

${ }^{10}$ J. H. Davies, I. A. Larkin, and E. V. Sukhourov, J. Appl. Phys. 77, 4504 (1995).

${ }^{11}$ J. Martorell, H. Wu, and D. W. L. Sprung, Phys. Rev. B 50, 17298 (1994).

${ }^{12}$ M. Chen and W. Porod, J. Appl. Phys. 78, 1 (1995).

${ }^{13}$ A. Kumar, S. E. Laux, and F. Stern, Phys. Rev. B 42, 5166 (1990).

${ }^{14}$ M. Stopa, Phys. Rev. B 48, 18340 (1993).

${ }^{15}$ M. Stopa, Phys. Rev. B 54, 13767 (1996).

${ }^{16}$ M. Macucci, K. Hess, and G. J. Iafrate, Phys. Rev. B 48, 17354 (1993).

${ }^{17}$ M. Macucci, K. Hess, and G. J. Iafrate, J. Appl. Phys. 77, 3267 (1995).

${ }^{18}$ M. Macucci, K. Hess, and G. J. Iafrate, Phys. Rev. B 55, R4879 (1997).

${ }^{19}$ J. C. Slater, Quantum Theory of Matter (McGraw-Hill, New York, 1968), and references therein.

${ }^{20}$ D. Pfannkuche, V. Gudmundsson, and P. A. Maksym, Phys. Rev. B 47, 2244 (1993). 